\title{
A Refined Propensity Account for GRW Theory
}

\author{
Lorenzo Lorenzetti ${ }^{1}$
}

Received: 24 October 2020 / Accepted: 22 February 2021 / Published online: 1 April 2021

(c) The Author(s) 2021

\begin{abstract}
Spontaneous collapse theories of quantum mechanics turn the usual Schrödinger equation into a stochastic dynamical law. In particular, in this paper I will focus on the GRW theory. Two philosophical issues that can be raised about GRW concern (a) the ontology of the theory, in particular the nature of the wave function and its role within the theory, and (b) the interpretation of the objective probabilities involved in the dynamics of the theory. During the last years, it has been claimed that we can take advantage of dispositional properties in order to develop an ontology for GRW theory, and also in order to ground the objective probabilities which are postulated by it. However, in this paper I will argue that the dispositional interpretations which have been discussed in the literature so far are either flawed orat best-incomplete. If we want to endorse a dispositional interpretation of GRW theory we thus need an extended account which specifies the precise nature of those properties and which makes also clear how they can correctly ground all the probabilities postulated by the theory. Thus, after having introduced several different kinds of probabilistic dispositions, I will try to fill the gap in the literature by proposing a novel and complete dispositional account of GRW, based on what I call spontaneous weighted multi-track propensities. I claim that such an account can satisfy both of our desiderata.
\end{abstract}

Keywords Collapse theories · GRW theory · Wave function · Dispositions · Propensities $\cdot$ Objective probabilities

Lorenzo Lorenzetti

lorenzo.lorenzetti@usi.ch

1 Università Della Svizzera Italiana, Via Buffi 13, 6900 Lugano, Switzerland 


\section{Introduction}

The Ghirardi-Rimini-Weber (GRW) theory ${ }^{1}$ is a spontaneous collapse theory of quantum mechanics which modifies the Schrödinger equation-i.e. the law which guides the temporal evolution of the wave function-to transform it into a (non-linear) stochastic dynamical law.

Several philosophical issues can be raised about collapse theories in quantum mechanics, and GRW theory has been the focus of the debate for the last two decades. Among them, two crucial questions concern (a) the ontology of GRW, in particular the nature of the wave function and its role within the theory, and (b) the interpretation of the probabilities involved in the theory. In this paper I will focus on the dispositional account of GRW. More precisely, it has been claimed-most notably by Dorato and Esfeld [15], Egg and Esfeld [16], Esfeld and Gisin [18] and Esfeld et al. [19] ${ }^{2}$ — that we can take advantage of dispositional properties in order to develop an ontology for GRW and also in order to ground the objective probabilities which are postulated by the theory. In addition, some authors-mainly Frigg and Hoefer [22] and Suárez [34] — have focused on providing an account of the probabilities in GRW as propensities - i.e. probabilistic dispositions. It should be stressed that, according to Frigg and Hoefer [22], the propensity theory of probability and the Humean account of chances are the only two viable interpretative option for the probabilities in GRW. ${ }^{3}$ Therefore, the propensity-dispositional account is particularly relevant in the context of GRW.

My aims in the present paper are the following. First of all, I will discuss the dispositional interpretations of GRW which are present in the literature. As I have remarked, one of their aims is to account for the objective probabilities implied by GRW in terms of probabilistic dispositions (or propensities). However, I will point out a crucial gap which is present in the account defended by Esfeld and his coauthors. In a nutshell, they merely define the dispositions in GRW as 'dispositions to localize spontaneously'. But the dispositions in GRW should be far more complex than that, as we will see from the technical presentation of the theory I will provide in the next section. If we want to endorse a dispositional interpretation of this theory-and of collapse theories in general-we thus need a complete account which specifies the full nature of those properties and which makes clear how they can ground the probabilities postulated by the theory. Frigg and Hoefer [22]—even though they do not focus on the metaphysics of the wave function in GRW-discuss all the possible interpretations of the probabilities in GRW, and give a few more details concerning the kinds of propensities that would be entailed by the theory. However, I will argue that their proposal is flawed and-in a way-incomplete. First of all, just like Esfeld and the others, they do not propose a detailed description of

\footnotetext{
1 The theory has been firstly presented by Ghirardi et al. [27]. In this paper I will focus on the nonrelativistic version of the theory.

${ }^{2}$ Even though it should be pointed out that Esfeld does not endorse dispositionalism anymore (see Esfeld and Deckert [17]).

3 See Childers [9] and Hájek [28] for an introduction to the propensity theory and to the Humean account of probabilities.
} 
the propensities entailed by the theory, but they present them rather informally. ${ }^{4}$ Secondly, they claim that the dynamics of GRW concerns two distinct coupled dispositions: I will suggest that this model seems to be at odds with the dynamics of the theory. Thirdly, and most importantly, their propensity model does not secure the mutual exclusivity of the different possible outcomes of the stochastic processsomething which is forced by the formalism of GRW. I shall show that also the account sketched by Suárez [34] faces the same objection. I believe that one crucial reason for which the latter important issue has been overlooked is that a rigorous treatment of the ontology of propensities in GRW is still missing in the literature. In fact-before putting forward these objections-I will introduce several different kinds of propensities, and I shall provide a detailed account of them by providing a formal characterization of each of them. Then, I will use that formal framework to formulate the objections mentioned above.

Therefore, taking stock of these objections, and having stressed the shortcomings of the existent dispositional approaches concerning GRW, I will propose a new dispositional account which can satisfy all of our desiderata. More precisely, I will single out a specific kind of probabilistic disposition among those that I have introduced, which is able to ground both the probabilities postulated by GRW through a single disposition: I call them spontaneous weighted multi-track propensities. Their form also secure that exactly one outcome is produced, and thus that the mutual exclusivity problem can be avoided. This novel account is meant to be a refined and extended version of the one proposed by Esfeld and his co-authors: it provides a more precise and explicit dispositional interpretation of the wave function within collapse theories and also a revised propensity interpretation of their probabilities. In this way I fill a crucial gap in the literature, since-as I have noticed-propensities are usually described in the context of GRW merely in informal terms. Moreover, another original feature of this paper will be to bring together the literature about the dispositional interpretation of the wave function in GRW and the one concerning the objective probabilities in GRW. As I have suggested, the former topic should not remain disconnected from the latter.

\section{A Brief Presentation of GRW Theory}

I shall now provide a very brief presentation of the basic principles of the GRW theory which we shall need for the continuation of the paper. I rely on the classic formulation given by Bassi and Ghirardi [5], pp. 41-42)—which provides a complete and technical presentation of the theory. ${ }^{5}$

Let us first define the usual Schrödinger equation:

\footnotetext{
${ }^{4}$ Even though it should be admitted that the focus of their paper are not propensities, but the Humean account of the probabilities in GRW.

5 See also Bassi and Ghirardi [4].
} 


$$
i \hbar \frac{\partial \psi}{\partial t}=H \psi
$$

where $H$ is the usual non-relativistic Schrödinger Hamiltonian. Then, Bassi and Ghirardi spell out the core of GRW via the following five points:

1. Each particle of a system of $n$ distinguishable particles experiences, with a mean rate $\lambda_{i}$, a sudden spontaneous localization process.

2. In the time interval between two successive spontaneous processes the system evolves according to the usual Schrödinger equation

3. The sudden spontaneous process is a localization described by:

$$
|\psi\rangle \stackrel{\text { localization }}{\longrightarrow} \frac{\left|\psi_{x}^{i}\right\rangle}{\|\left|\psi_{x}^{i}\right\rangle \|}
$$

where $\left|\psi_{x}^{i}\right\rangle=L_{x}^{i}|\psi\rangle . L_{x}^{i}$ is a norm-reducing, positive, self-adjoint, linear operator in the $n$-particle Hilbert space $\mathrm{H}$, representing the localization of particle $i$ around the point $\boldsymbol{x}$

4. The probability density for the occurrence of a localization at point $\boldsymbol{x}$ is assumed to be:

$$
P_{i}(x)=\|\left|\psi_{x}^{i}\right\rangle \|^{2}
$$

This requires that:

$$
\int d^{3} x\left[L_{x}^{i}\right]^{2}=1
$$

5. The localization operators $L_{x}^{i}$ have been chosen to have the form:

$$
L_{x}^{i}=\left(\frac{\alpha}{\pi}\right)^{3 / 4} e^{-\frac{\alpha}{2}\left(q_{i}-x\right)^{2}}
$$

$q_{i}$ being the position operator for particle $i$ ([5], pp. 41-42)

The value of the parameters $\lambda$ (for a single particle) and $\alpha$ are carefully chosen as follows:

$$
\begin{gathered}
\lambda \cong 10^{-16} s^{-1} \\
1 / \sqrt{\alpha} \cong 10^{-7} \mathrm{~m}
\end{gathered}
$$

The parameter $1 / \sqrt{\alpha}$ is a distance and it measures the distance between the two peaks of localization of the two superposed elements, when the collapse happens and the superposition is eliminated. On the other hand, the value of $\lambda$ entails that a single particle system is localized once every $10^{8}-10^{9}$ years. This means that a single proton can remain in a superposed state for a very long time. By contrast, 
macroscopic systems behave in a different way. The relation between the frequencies of the macro-systems and the ones of the micro-systems is expressed by the following equation:

$$
\lambda_{\text {macro }}=\sum_{k=1}^{n} \lambda_{k}
$$

The theory assumes that all the values $\lambda_{k}$ are equal (i.e. they all correspond to $\lambda \cong 10^{-16} s^{-1}$ ). Thus, considering a system composed by $n$ microscopic constituents, we have that $\lambda_{\text {macro }}=n \lambda$. Consider a macroscopic system made up of $10^{23}$ microscopic particles. Then, $\lambda_{\text {macro }} \cong 10^{7} s^{-1}$. This explains why we do not have superposed states as results of measurement processes. ${ }^{6}$

The occurrence of hits in GRW is a Poisson process, i.e. a process which represent the number of occurrences of a type of event which take place during an interval of time $\tau$. The probabilities which characterize Poisson distributions are represented as follows:

$$
p(n=m)=\frac{e^{-\lambda \tau}(\lambda \tau)^{m}}{m !},
$$

where $p(n=m)$ is the probability that the number of events $n$ occurring during the time interval $\tau$ takes value $m=0,1,2, \ldots$ and $\lambda$ is the parameter of the distribution. ${ }^{7}$ $\lambda$ can be considered as the average number of events per unit time. Thus, we can say that "the probability of an event occuring during the infinitesimal interval $d t$ is $\lambda d t$ " [22], p. 373. As we have seen, GRW sets the value of $\lambda$ to be $\lambda \cong 10^{-16} s^{-1}$. Two peculiar features of Poisson processes are that the rate $\lambda$ is constant over time and that each event is stochastically independent to the other events in the Poisson process.

We can now complete the presentation of GRW theory by introducing the central stochastic equation which substitutes the Schrödinger equation-keeping in mind that, within GRW, the reduction mechanism transforms pure states into statistical mixtures. Following Bassi and Ghirardi ([5], p. 43) we can indeed express the hitting process for a single particle, whose wave function $|\psi\rangle$ is transformed into $\left|\psi_{x}\right\rangle$, as follows:

$$
|\psi\rangle\left\langle\psi\left|=\int d^{3} x P(x) \frac{|\psi\rangle\langle\psi|}{\|\left|\psi_{x}\right\rangle \|^{2}}=\int d^{3} x L_{x}^{i}\right| \psi\right\rangle\langle\psi| L_{x}^{i} \equiv T[|\psi\rangle\langle\psi|]
$$

Bassi and Ghirardi then show how to derive the evolution equation for $\rho(t)$ during a time interval $d t$. As we have seen, the localization process is Poissonian and specifies a probability $\lambda d t$ for the occurrence of a hit during the interval $d t$-in which $\rho$ is transformed to $T[\rho]$-and, conversely, a probability $1-\lambda d t$ for the non-occurrence of hits. The evolution equation for $\rho(t)$ can thus be stated as follows:

\footnotetext{
${ }^{6}$ See Ghirardi ([25], Ch. 16) for a detailed introduction of this process.

7 Cf. Frigg ([21], p. 267).
} 


$$
\begin{gathered}
\rho(t+d t)=(1-\lambda d t)\left[\rho(t)-\frac{i}{\hbar}[H, \rho(t)] d t\right]+\lambda d t T[\rho(t)] \\
\text { i.e. } \frac{d}{d t} \rho(t)=-\frac{i}{\hbar}[H, \rho(t)]-\lambda(\rho(t)-T[\rho(t)]) .
\end{gathered}
$$

This presentation gives us a minimal overview of the central core of GRW theory. However, this is everything which we will need in order to discuss the main topics of the present paper. Before moving on, however, I want to make a couple of points. The first issue I want to raise is the role of probabilities within the theory. In GRW, we have described two probabilistic aspects: (a) the 'sudden spontaneous localization process' happens with a given frequency, and thus the system has only a small probability per unit time of undergoing a hit, and (b) the localization point is chosen randomly or, better, is determined by a specific probability density in accordance to the predictions of Born's rule. Moreover, it should be pointed out that those probabilities must be objective. In other words, any subjective reading of the probabilities in collapse theories is ruled out. ${ }^{9}$ This is made clear especially by the fact that all collapse theories are realist approaches to quantum theory which do not depend on the existence of observers who perform the measurements. In the words of Ghirardi ([25], p. 409): “no observer carries out any measurement: nature itself (Einstein's God?) chooses to induce such a process according to random choices but with precise probabilities".

However, before we delve further into this topic, we should first provide a clearer picture concerning the ontology of the theory. Viz., we should explain what there is in the world, according to collapse theories. Until now, we have only discussed about the wave function, and we have considered it as the complete description of any quantum system. However, it is nowadays widely believed ${ }^{10}$ that the wave function is not everything there is according to collapse theories: we should specify also a-equally fundamental-primitive ontology.

\section{Primitive Ontologies for the GRW Theory}

Several physicists and philosophers of science share the opinion that the formal rules presented so far, which are the ones used to make predictions, are not all there is to say about GRW. According to Bell, ${ }^{11}$ and according to Bassi and Ghirardi, ${ }^{12}$ we should specify also a fundamental primitive ontology for collapse theoriesbesides the purely formal aspects of the theories - to give an account of the fundamental constituents of reality. This issue has been presented in the clearest way

\footnotetext{
${ }^{8}$ Cf. Bassi and Ghirardi ([5], p. 43).

9 See also Frigg and Hoefer ([22], p. 376) on this point.

10 See Clifton and Monton [12] and Albert [1, 2] for a different opinion.

11 See Bell [6].

12 See Bassi and Ghirardi [4].
} 
in a landmark paper by Allori et al. [3], where the authors present the two main alternative ontologies for collapse theories (focusing on GRW): the flash ontology (GRWf) and the mass density ontology (GRWm). ${ }^{13}$ In this section I will present these two alternatives, focusing on the relation between these ontologies and the wave function.

GRWm was originally proposed by Ghirardi et al. [26]. Within GRWm, the primitive ontology is constituted by a matter-density field of variables $m(x, t)$ ranging over points in three-dimensional space at times $t$, defined as follows ([3], p. 359):

$$
m(x, t)=\sum_{i=1}^{N} m_{i} \int_{\mathbb{R}^{3 N}} d q_{1} \ldots d q_{n} \delta\left(q_{i}-x\right)\left|\psi\left(q_{1}, \ldots, q_{N}, t\right)\right|^{2} .
$$

Essentially, according to GRWm, what exists at the fundamental level is just a distribution of mass in three-dimensional space whose behaviour is determined by the wave function in accordance with the stochastic dynamics which has been presented in the last section.

On the other hand, GRWf provides a completely different ontology. One of the first formulations of this ontology has been advanced by Bell [6]. In the words of Allori et al., according to this account 'the primitive ontology is given by 'events' in space-time called flashes, mathematically described by [discrete] points in space-time" ([3], p. 360). Similarly to GRWm, in GRWf the spatiotemporal locations of these flashes is determined by the history of the wave function: "Every flash corresponds to one of the spontaneous collapses of the wave function, and its space-time location is just the space-time location of that collapse" (Ibidem).

After having presented the two alternative accounts for the primitive ontology of GRW, we should notice the following. Within both accounts, the primitive ontology is not the only thing which matters: on the contrary, the wave function plays a crucial role. This is strongly stressed by Allori et al. [3]. They are in fact very clear in pointing out that, within this picture, the wave function tells matter how to move, viz. it governs the primitive ontology. That is, the wave function has a remarkable nomological character-at least prima facie.

The presentation of GRW we have brought out so far has shown very clearly two distinct aspects which deserve careful philosophical investigation. First, we should provide an interpretation of the intrinsic probabilities of the theory. To do so, we should explain what grounds them. Second, given the important role which is played by the wave function, we should provide an account of its nature.

In recent years, the literature concerning GRW - and, in general, primitive ontology in quantum mechanics - has focused mainly on the latter issue. ${ }^{14}$ On the other hand, some other authors_-most notably Suárez [34] and Frigg and Hoefer [22] have focused on the former issue. In contrast, my aim for the rest of this paper will be different: I will discuss a metaphysical account that can provide a unified metaphysical picture which gives answers to the main questions arising in each of the

\footnotetext{
13 Or, alternatively, 'matter' density.

14 See for example Belot [7], Esfeld et al. [19] or Chen [10] for an overview.
} 
areas. In particular, I will focus on the dispositional account of GRW developed by Dorato and Esfeld [15] and Egg and Esfeld [16]. As I will argue, that account is not satisfactory as it stands and should be amended.

\section{Dorato and Esfeld on GRW as an Ontology of Dispositions}

Dorato and Esfeld [15] argue that a plausible way to interpret the spontaneous localizations that are implied by spontaneous collapse theories is by claiming that according to GRW “non-massless micro-systems possess a disposition for spontaneous localization" ([15], p. 43). This view has been discussed and defended also by Suárez [34], Dorato [14], Egg and Esfeld [16], Esfeld and Gisin [18] and Esfeld et al. [19].

This kind of disposition differs from the standard definition of dispositional property in two important ways. Let's look at the classical conditional analysis of (deterministic) dispositions ${ }^{15}$ :

$\mathrm{F}$ is a disposition [or dispositional property] iff there are an associated stimulus condition and manifestation such that, necessarily, $\mathrm{x}$ has $\mathrm{F}$ only if $\mathrm{x}$ would produce the manifestation $M$ if it were in the stimulus condition $S$ (Choi and Fara [11]).

The dispositions within GRW, on the other hand, do not need to be in any particular stimulus condition to be activated; instead, they manifest themselves spontaneously, in the sense that their manifestations are independent of any interaction. Moreover, those dispositions can be regarded as propensities. In fact, probability values are attached to their manifestations. In other words, it is not true that once those dispositions are triggered, a single unique manifestation $\mathrm{M}$ is produced with certainty. There are different ways to spell out the notion of propensity, and I will say more about these concepts in Sect. 5 .

Let us consider now more carefully what is the precise role of those dispositions within the ontology of GRW, for Dorato and Esfeld. First of all, according to them the fundamental ontology is constitued by the entities composing the primitive ontology of the theory, i.e. either by "flashes" (in GRWf) or by the density of "stuff" (in GRWm). Then, consider a superposed state like the following:

$$
|\psi\rangle=\frac{1}{\sqrt{2}}|A\rangle+\frac{1}{\sqrt{2}}|B\rangle .
$$

According to them, "the disposition for spontaneous localization [...] is the essence of such spatially superposed states, since the latter, according to GRW, are to be regarded as intrinsically unstable" (Dorato and Esfeld [15], p. 44). For a physical system to be in a superposed state like $|\psi\rangle$ means to have a certain probability to localize spontaneously either in region A or in region B-according to GRW. Thus,

15 See also Bird [8] for a classical account of dispositions. 
according to Dorato and Esfeld, if a physical system is in such state, then it possesses a disposition to localize: "the wave function is a mathematical symbol essentially referring to such propensities" (Ibid.).

Summing up their position, Dorato and Esfeld claim that both the primitive ontology and the wave function-qua disposition - are fundamental. In particular, following Allori et al. [3], they believe that "the role of the wave function is to provide an algorithm that guides the evolution of the physical system" (Ibidem, p. 47). On the one hand, in the case of GRWf the flashes are the manifestations of those dispositions to localize. ${ }^{16}$ On the other hand, concerning GRWm, the dispositions govern the evolution of the mass density field. The latter view is shared also by Egg and Esfeld [16].

We should now notice that the dispositional view defended by Dorato and Esfeld [15], Egg and Esfeld [16] is meant to give also an account of objective probabilities in GRW in term of propensities. ${ }^{17}$ They claim that by attributing intrinsic dispositions to the physical systems governed by GRW we are able to attribute singlecase probabilities, in the sense that we are able to say that single systems possess an objective probability to localize. Those probabilities do not derive from the frequencies that we register: on the contrary, the frequencies are the manifestation of the propensities to localize. In other words, according to the dispositional account, the objective probabilities in GRW are grounded on the nature of those dispositional properties which characterize superposed states.

The problem with their account is that they merely say that, according to GRW, physical systems are characterized by "dispositions to localize spontaneously". I take this to be simply a rough proposal for an account of GRW in terms of dispositions, and not a fully-fledged account, of course. If our aim is also to ground the objective probabilities of GRW on propensities, then we need to specify more precisely what the metaphysical structure of those properties is and how they are able to produce the exact probabilities of the theory. ${ }^{18}$ This will be the main topic of the rest of the paper.

\footnotetext{
$\overline{16}$ See also Esfeld and Gisin ([18], p. 252) for a brief discussion of GRWf as an ontology of dispositions exactly alike the one defended by Dorato and Esfeld [15].

17 Arguably, it is easy to show that, when considered in the specific context of collapse theories in quantum mechanics, the propensity theory of probability does not face the usual challenges that are raised against it (e.g. the reference class problem). See Frigg and Hoefer ([22], p. 384-5) for a defence of this claim.

18 Placek [32] develops a detailed causal model for the probabilities assigned to the localizations of flashes within GRWf (and the relativistic version of GRWf). However-unlike the account I am going to present - his proposal is limited to GRWf and in particular to the probabilities linked to the flashes distribution. In other words, he considers only one of the two probabilistic terms of GRW. But most importantly, his account is based on causal probabilistic spaces and branching-time models, and not on a metaphysics of dispositional properties - at least in the sense in which dispositions have been discussed in the literature (i.e. as properties identified via counterfactual conditionals). On the contrary, my current aim is to craft a dispositional account of GRW which accommodates GRW probabilities within the standard notion of dispositional property.
} 


\section{A Detailed Topology of Propensities}

Some attempts have been made to account for the objective probabilities in GRW in terms of dispositions, quite independently from the issue concerning the physical meaning of the wave function within the theory. However, these accounts are barely sketches of theories, and moreover I shall argue that they all faces problematic consequences. Thus, a complete and adequate account is needed. In this section I will firstly propose a novel detailed account of propensities in general, which can account for different kinds of propensities. Secondly, I shall introduce the proposals given by Frigg and Hoefer [22] and Suárez [34] for a propensity theory of GRW. Then, relying on the propensity account that I am going to propose, I will argue that-once they are carefully formulated-both their proposals are problematic. Finally, I shall put forward a novel propensity theory of GRW which can overcome the problems faced by the other theories.

The propensity account of probabilities traces back to Popper [33], but many different versions of this theory have been defended throughout the years (see for example Fetzer [20], Miller [31], Gillies [24] and Mellor [29, 30]). In the following, I will try to spell out a specific version of the propensity theory, tailored to account for the probabilities in GRW.

Summing up what I have pointed out so far about propensities, we can say thataccording to the propensity account_probability statements are made true by irreducibly dispositional properties possessed by objects. This means that probability is an objective feature of the world. As I have claimed in Sect. 2, the probabilities in GRW are required to be objective, and thus the propensity theory can easily accomodate this desideratum. Moreover, as we have noticed, the propensities in GRW are single-case in the following sense: "A single-case propensity theory is one in which propensities are regarded as propensities to produce a particular result on a specific occasion" (Gillies [24], p. 822). That is, the propensities in question are not linked to ensembles or to the repetition of trials. On the contrary, they are dispositional properties possessed by single physical systems which ground probabilities of a single particular outcome, tied to the single object which instantiates them.

First of all, I will now introduce a distinction within the category of propensities. We can indeed distinguish between what I call single-track and multi-track propensities. I define single-track propensities as follows, adopting Suárez's ([34], p. 430) definition of propensities, slighlty reformulated:

Single-track propensity: If object $O$ possesses single-track propensity $P$ with manifestation $M$ then: were $O$ to be in the appropriate circumstance $C$, it would $M$ with probability $p(0 \leq p \leq 1)$.

They are called single-track propensities because they are defined by a single (counterfactual) conditional which links the triggering condition $\mathrm{C}$ with a unique manifestation event M. However, we can easily modify this definition to account also for spontanously triggered dispositions:

Spontaneous single-track propensity: If object $O$ possesses spontaneous single-track propensity $P_{S}$ with manifestation $M$ then: were $O$ to instanti- 
ate $P_{S}$ during an infinitesimal interval of time $d t$, during $d t$ it would $M$ with probability $\lambda d t(0 \leq \lambda d t \leq 1)$.

In other words, the 'triggering condition' of a spontanous single-track propensity is simply its existence. They are defined by an unique manifestation $\mathrm{M}$, and for a given interval of time the bearer of that kind of propensity can either manifest $\mathrm{M}$ or not manifest $\mathrm{M}$.

In addition to single-track propensities, I introduce the notion of multi-track propensities $^{19}$ :

Multi-track propensities: If object $O$ possesses probabilistic disposition $D$ with set of mutually exclusive manifestations $\left\{M_{1}, \ldots, M_{n} \mid n \geq 2\right\}$, then: were $O$ in the condition $\Sigma$, it would produce exactly one manifestation $M_{i}$ from the set of manifestations $\left\{M_{1}, \ldots, M_{n} \mid n \geq 2\right\}$.

Notice that they are called 'multi-track' because the same stimulus condition can lead to different manifestations. Moreover, when they are triggered, at least one manifestation is produced, ${ }^{20}$ even though it is randomly selected. It should be stressed, however, that this kind of propensities presents a possible drawback. In fact, from the defintion above it follows that multi-track propensities generate their manifestation totally randomly. But there may be contexts-like in GRW, as we will seewhere the manifestation is picked randomly from the set of possible manifestations but, at the same time, accordingly to a probability distribution. That is, we want to be able to assign different probabilities to the distinct outcomes of propensities.

To solve this problem, firstly we can define a probability mass function which assign different probabilities to the members of the set $\left\{M_{1}, \ldots, M_{n} \mid n \geq 2\right\}$ as follows ${ }^{21}$.

The probability mass function $p$ of a discrete random variable $X$ is the function $p: \mathbb{R} \rightarrow[0,1]$, defined by

$$
p(M)=P(X=M) \text { for } M_{1}, \ldots, M_{n} .
$$

If $X$ is a discrete random variable that takes on the values $M_{1}, M_{2}, \ldots$, then

$$
p\left(M_{i}\right)>0, p\left(M_{1}\right)+p\left(M_{2}\right)+\cdots=1, \text { and } p(M)=0 \text { for all other } M .
$$

The idea is thus to embed this probability function into the definition of multitrack propensity. Let us label this revised version of multi-track propensities as weighted multi-track propensities:

Weighted multi-track propensity: If object $O$ possesses weighted multitrack propensity $D_{W}$, with set of mutually exclusive manifestations

\footnotetext{
${ }^{19}$ Cf. Gebharter and Fischer ([23], p. 3).

${ }^{20}$ Notice here that single-track propensities can actually be considered as very special cases of multitrack propensities, where the set of (mutually exclusive) possible manifestations is composed by $\{M$ or not- $M$ \}. However, for simplicity, we will stick to the formulation stated above.

${ }^{21}$ Cf. Dekking et al. ([13], p. 43).
} 
$\left\{M_{1}, \ldots, M_{n} \mid n \geq 2\right\}$, where a specific value $p\left(M_{i}\right)$ given by the probability mass density $p$, with $0 \leq p\left(M_{i}\right) \leq 1$, is assigned to each member $M_{i}$ of that set and $p\left(M_{1}\right)+\cdots+p\left(M_{n}\right)=1$, then: were $O$ in the condition $\Sigma$, it would produce exactly one manifestation $M_{i}$ from the set of manifestations $\left\{M_{1}, \ldots, M_{n} \mid n \geq 2\right\}$, where the probability of producing $M_{i}$ is given by $p\left(M_{i}\right)$.

Moreover, just as for single-track propensities, multi-track propensities too can be spontanouesly triggered. We just have to specify a spontaneous triggering probability $\lambda d t$ over the appropriate infinitesimal interval of time $d t$, which fixes the probability for the spontaneous activation of the disposition during that interval. We can thus define the notion of spontaneous weighted multi-track propensity:

Spontaneous weighted multi-track propensity: If object $O$ possesses spontanous weighted multi-track propensity $D_{S W}$ during an infinitesimal interval of time $d t$, with set of mutually exclusive manifestations $\left\{M_{1}, \ldots, M_{n} \mid n \geq 2\right\}$, where a specific value $p\left(M_{i}\right)$ given by the probability mass density $p$, with $0 \leq p\left(M_{i}\right) \leq 1$, is assigned to each member $M_{i}$ of that set and $p\left(M_{1}\right)+\cdots+p\left(M_{n}\right)=1$, then: were $O$ to possess $D_{S W}$ during $d t$, during $d t$ it would produce exactly one manifestation $M_{i}$ from the set of manifestations $\left\{M_{1}, \ldots, M_{n} \mid n \geq 2\right\}$ - where the probability of producing $M_{i}$ is given by $p\left(M_{i}\right)$ —with probability $\lambda d t$.

To recap, we have now defined two distinct notions of propensities: the former specify a kind of disposition which has merely a certain degree of probability to produce its unique manifestation; the latter, on the contrary, produce with probability $p=1 \mathrm{a}$ manifestation, but that manifestation is chosen randomly-in accordance with a precise probability distribution - from a set of possible manifestations. In addition, both these kinds of propensities can produce their manifestations either given a certain stimulus condition or spontaneously. They both implement probabilities within their definitions, but they do that in slighly different ways: while single-track propensities can accommodate at most one probabilistic term, multi-track propensities-due to their manifestation selection step — can ground up to two probabilistic processes. I will argue that spontaneous weighted multi-track propensities are what we need to account for the probabilities in GRW.

As I made clear in Sect. 2, there are two fundamental objective probabilities within GRW: the probability associated to the Poissonian hitting process, linked with the frequency value $\lambda \cong 10^{-16} s^{-1}$, and the probability density $P_{i}(x)=\|\left|\psi_{x}^{i}\right\rangle \|^{2}$ assigned to the localization process. Thus, we should account for both probabilistic processes. $^{22}$

\footnotetext{
${ }^{22}$ The belief that GRW incorporates two distinct fundamental probabilities, and that we should provide a philosophical account for both, is shared also by Timpson ([35], p. 208) and by Frigg and Hoefer ([22], p. 384).
} 


\section{Propensities in GRW}

Now, before I advance my proposal for a dispositional account of GRW, I will analyse Frigg and Hoefer's and Suárez's proposals in the light of the different types of propensities that I have introduced so far, in order to show which problems emerge from their accounts once we spell it out in a clearer form.

Starting with Frigg and Hoefer [22], they have proposed a reading of GRW probabilities in terms of propensities, which is slightly more detailed than Dorato and Esfeld's one. They argue that, according to this interpretation of GRW, physical systems possess two kinds of single-case propensities, which ground the corresponding random processes and their respective probabilities. They also highlight the fact that those two processes are coupled, because one provides the trigger for the other. On the one hand, they claim that the occurrence of hits is grounded by spontaneous propensities:

The occurrence of a localization [...] does not seem to [have] a triggering condition [...] There is a chance of $\lambda d t$ for each elementary constituent to decay during $d t[\ldots]$. Hence [this random process] can be understood on the basis of the single case propensity view. (Frigg and Hoefer [22], p. 384).

On the other hand, regarding the localization processes, they claim that:

It seems natural to say that for every possible localization event $H_{x}$ the wave function has a (single case) propensity to undergo this particular localization. (Ibid.).

However, they do not give further details about the structure of their propensity reading of GRW. In addition, I am going to show that-once we formalize this proposal-some issues emerge.

First of all, as we have seen, they claim that the dynamics of GRW embeds two coupled random processes, and that a propensity theory of probability should ground the probabilities assigned to those processes onto two kinds of propensities. Indeed, they seem to distinguish between those propensities which ground the hitting processes and those that underlie the localization processes.

Considering the first of the two quotes above, it seems that they want to characterize the objective chances embedded within the random hitting processes as spontaneous single-track propensities. Remember that I have defined these as follows: "If object $O$ possesses spontaneous single-track propensity $P_{S}$ with manifestation $M$ then: were $O$ to instantiate $P_{S}$ during an infinitesimal interval of time $d t$, during $d t$ it would $M$ with probability $\lambda d t(0 \leq \lambda d t \leq 1)$.". Thus, we can say that hitting processes are caused by the (spontaneous) triggering of spontaneous single-track propensities possessed by physical systems, whose manifestation $M$ is the occurrence of the hit and whose probability $\lambda d t$ is determined by the value of the parameter $\lambda$ postulated by GRW. Those propensities determine thus the Poisson distributions defined by the theory.

Passing now to the second kind of random processes within GRW-i.e. the localization processes-let us consider as our case study the simple superposed state $|\psi\rangle$ 
of Sect. 3. According to Frigg and Hoefer, the occurrence of the first random process - the hit-triggers the localization process $|\psi\rangle \stackrel{\text { loc. }}{\longrightarrow} \frac{\left|\psi_{x}^{i}\right\rangle}{\|\left|\psi_{x}^{i}\right\rangle \|}$. Thus, the quantum state $|\psi\rangle$ localizes either in region $\mathrm{A}$ or in region $\mathrm{B}$, with a probability given by $P_{i}(x)=\|\left|\psi_{x}^{i}\right\rangle \|^{2}$, where $x$ is where the localization takes place. Concerning the quantum system in our example, the same probability $P_{i}(x)=0.5$ is assigned to both the locations.

As I have already remarked, they claim that the most natural way to conceive this process is "to say that for every possible localization event $H_{x}$ the wave function has a (single case) propensity to undergo this particular localization". This seems to suggest to model those propensities as simple single-track propensities ("If object $O$ possesses single-track propensity $P$ with manifestation $M$ then: were $O$ to be in the appropriate circumstance $C$, it would $M$ with probability $p(0 \leq p \leq 1)$."). More precisely, each system possesses a set of individual and distinct single-track propensities, one for every localization event $H_{x}$ : the appropriate circumstances are constituted by the occurrence of the hits, the localization events are the manifestations of those propensities-i.e. they are caused by them-and their probabilities are fixed by the probability function $P_{i}(x)$.

To recap, the propensity account of the random processes in GRW proposed by Frigg and Hoefer can be stated as follows: spontaneous single-track propensities produce hits which, in turn, trigger single-track propensities whose manifestations are the localizations of the wave function.

In the following, I shall point out why I find this account unsatisfactory. First of all, I shall move what I consider to be a decisive objection against this specific propensity view. That is, I will claim that the model sketched by Frigg and Hoefer fails to secure the mutual exclusivity of the different possible localization events. Secondly, I will add some considerations concerning the fact that this account-as I have formalized it-embeds two distinct and coupled probabilistic dispositions.

Starting from the first point, we can raise the following objection against Frigg and Hoefer's account of dispositions to localize. Consider the quantum state $|\psi\rangle$ mentioned above. According to their account, the quantum system $S$ described by $|\psi\rangle$ possesses two distinct propensities $P_{1}$ and $P_{2}$ :

(1) $S$ possesses the single-track propensity $P_{1}$ such that: were there an occurrence of a hit, $S$ would localize in the region A with probability $p=0.5$

(2) $S$ possesses the single-track propensity $P_{2}$ such that: were there an occurrence of a hit, $S$ would localize in the region B with probability $p=0.5$

Recall that localization events are produced by dispositions to localize. Now, suppose that system $S$ undergoes a hit. Therefore, by the definition of single-track propensities $P_{1}$ and $P_{2}$, both those dispositions would be triggered. Each disposition would produce its manifestation - the localization event-only probabilistically. But, notice that the structure of those propensities would entail an unwelcomed result. In fact, a total of four combinations of outcomes are consistent with the nature of $P_{1}$ and $P_{2}$ so defined: in two of those occasions, only one of the two localization 
event takes place, but there would be also one situation where no localization is produced and one another where two localization events are displayed. But, of course, the last two situations are forbidden by the theory. In other words, this model fails to account for the mutual exclusivity of the two localization events.

I pass now to the second consideration that I want to point out concerning Frigg and Hoefer's proposal. Looking at the formalism of the theory, the localization process postulated by GRW is governed by a single stochastic equation and is instantaneous and spontaneous. Because of this, it seems odd to say that the dynamics of GRW concerns two distinct coupled dispositions, one of which provides the triggering conditions for the other. The reason is the following.

Let's assume that the manifestation of a disposition corresponds to a physical event. Then, according to the two-propensities model, we would have a first process-grounded by a spontaneous single-track propensity-whose manifestation is the hit. The hit, therefore, would be a physical event, and it would trigger the activation of a disposition to localize (let's set aside here the problem of mutual exclusivity). Thus, the manifestation of the second disposition would correspond to the physical event which we actually measure, i.e. the localization of the system in a single region. Given this description, two puzzling aspects arise.

Firstly, it should be pointed out that the hit is not a physical event at all. The hit simply represent mathematically the fact that the wave function collapse. However, it would not make much sense to say that this description corresponds to a physical event, since it would mean that there is a collapse but nowhere in spacetime, given that-by definition - the actual localization is produced by the second disposition. In addition, we also have no reason to claim that only the manifestation which has physical meaning is the one of the second disposition, since that would commit one to say that two radically different kinds of dispositions are at play here, such that the nature of their manifestations is different. Secondly, and relatedly, this model seems to suggest that there is a temporal succession between the hit and the localization. However, this is not what GRW postulates. The process of localization is, on the contrary, instantaneous. The most natural interpretation would be therefore that the disposition underlying this process is a single propensity which has as its unique manifestation a spatially-localized event.

Admittedly, the strength of this objection depends on one's view about dispositions. However, I believe that here I have at least made the case for believing that-all things being equal-we should prefer a propensity account of GRW which grounds the dynamics on the theory on one single disposition only, in place of a two-propensity model.

Let's consider now Suárez's [34] proposal. Similarly to Frigg and Hoefer, he has sketched a possible interpretation of the probabilities within GRW in terms of probabilistic dispositions. He claims that:

The localization process [of GRW] is at the very least compatible with the assumption that each quantum particle has an irreducible disposition to localize in an area given by $d$ with frequency $f$. [...] The dispositions that according to GRW each particle has to spontaneously reduce upon a region $x$ of area $d$ are propensities. (Suárez [34], p. 433) 
Here the author is explicitly using the term 'propensities' to denote what I have called 'single-track propensities'. But he also stresses that the propensities within GRW cannot have that exact form, since they are spontaneously triggered. At that point, however, Suárez does not propose a revised definition of propensities which can account for this feature. Thus, in order to spell out more precisely the account he is sketching, I shall take spontaneous single-track propensities as a charitable formulation of the kind of dispositions he is referring to.

Given what he says in the quote aforementioned, it seems natural to interpret his account as postulating that in GRW each 'quantum particle' possesses a set of spontaneous single-track propensities. Each of those propensities is characterized by its disposition to localize the particle in a different region of space. ${ }^{23}$ In other words, the particle possesses a propensity for every region in which it can be localized. Taking again the superposed state $|\psi\rangle$ of Sect. 3 as our example, in that case the system would possess both one propensity to localize in region A and one propensity to localize in region B. It is now quite easy to notice that a very similar objection to the one which I have raised against Frigg and Hoefer's account can be proposed in this context as well. That is, since here we are talking about two distinct dispositions, nothing in the metaphysical structure of the account forbids the two spontaneous propensities to manifest together at the same time. Given that they are-so to say-uncorrelated, it is possible for them to spontaneously manifest together. This scenario is of course very unlikely, but its mere possibility is sufficient to threaten the account. Indeed, just as we have seen concerning Frigg and Hoefer's account, a simultaneous manifestation is forbidden by the physics of GRW, and thus any metaphysical account of the theory have to rule out this scenario categorically.

To recap, starting from the reasons that I have put forward for rejecting the account proposed by Frigg and Hoefer and by Suárez, we can formulate a crucial desideratum that any propensity theory of GRW has to satisfy. That is, it should secure the mutual exclusivity of the possible localization events - and that exactly one of them is produced. Then, a second minor desideratum is arguably to avoid postulating a two-propensity model similar to the one envisaged within Frigg and Hoefer's proposal.

I claim that if we adopt an account of GRW probabilities in term of spontaneous weighted multi-track propensities we can easily satisfy these desiderata. More precisely, we can claim that physical systems ${ }^{24}$ instantiate a single disposition-a spontaneous weighted multi-track propensity—which grounds both the random processes featured in GRW at once. On the one hand, the 'spontaneous' nature of those

\footnotetext{
${ }^{23}$ Notice that in this case the probability $\lambda d t$ takes into account both the probability that a spontaneous hit takes place and the probability - given by Born's rule - that the localization happens in that specific region.

${ }^{24}$ Like the other authors working on this topic, I remain neutral concerning the exact bearers of the dispositions. Within GRWm we can plausibly say that the dispositions are possessed by the primitive ontology-i.e. the matter. Within GRWf, on the other hand, the issue is more tricky. However, I want to stress that this particular 'ambiguity' concerning what physical systems are and what are the bearers of the dispositions is not a peculiarity of this account but, rather, it is shared by any of the dispositional accounts which I have mentioned before.
} 
dispositions, which is formally represented by the disposition's probability to be self-triggered during a specific interval of time, grounds the frequency $\lambda$ for the hits to occur. On the other hand, those dispositions are probabilistic also because-when triggerred-they produce a "random" manifestation from a set of possible manifestations. However, since they are weighted multi-track propensities, by definition that outcome will not be completely casual. On the contrary, the very nature of those dispositions is to assign a distribution of probabilities over the set of their possible outcomes. And this is what grounds the objective probabilities linked to the localization process-i.e. the Born's rule predictions. More precisely, the set of mutually exclusive manifestations of these propensities is the set of all the possible localizations events, while the probabilities $p\left(M_{i}\right)$ for the localization events are the ones assigned by the function $P_{i}(x)=\|\left|\psi_{x}^{i}\right\rangle \|^{2}$.

In sum, I claim that this propensity account for GRW fares better than its alternatives. First and foremost, thanks to its appeal to multi-track propensities, it is the only one-among the ones discussed-which secures the mutual exclusivity of the different localization events, thereby avoiding the objection which I have put forward in this section. I take this to be the most decisive advantage of this view. Secondly, I claim that —all this being equal — this account should be preferred, since it is more simple and ontologically parsimonious than the alternative ones suggested above. In fact, it postulates a single multi-track propensity to ground the localization process and the probabilities described by GRW. Thus, it is neither committed to the existence of whole sets of dispositions (one for each possible manifestation), unlike the two accounts which I have assessed, nor to the existence of two distinct kinds of dispositions, unlike Frigg and Hoefer's view. In this sense, the account I proposed here is more parsimonious both in the number and in the kinds of the propensities that it assumes. Finally, it avoids the second of the two objections that I have moved against the account sketched by Frigg and Hoefer. In fact, within my account, the collapse and the localization are simultaneously realized by the same manifestation: once the spontaneous weighted multi-track propensity is (spontaneously) activated, it simply produces the localization of the system as its unique manifestation. As I said before, this is not by itself a decise point in favour of this proposal, and one could still insist that in practice the two-propensity model by Frigg and Hoefer does not differ substantially from my account, in this respect. However, ceteris paribus, I believe that a simpler model is to be preferred.

In conclusion, my proposal for a dispositionalist ontology of GRW is the following. Physical systems (in the appropriate conditions) instantiate spontaneous weighted multi-track propensities, which grounds the two probabilistic terms $\lambda$ and $P_{i}(x)$. The fundamental ontology of the world is composed by those dispositions, in addition to the primitive ontology of GRW. The primitive ontology is thus governed by them. Moreover, I side with Dorato and Esfeld [15] and Egg and Esfeld [16] concerning their interpretation of the wave function within GRW. Indeed, we can say that wave function "is a mathematical symbol essentially referring to such propensities". The main aim of this paper has been to refine their account, by showing how to spell out a complete dispositional interpretation of the wave function in GRW which can also ground the objective probabilities postulated by the theory. Of 
course, in this paper I have not argued in favour of the dispositionalist view per se. However, what I want to urge is that, if one wants to endorse a dispositionalist view of GRW, this would be the best account available.

\section{Conclusion}

During the last years, dispositionalism has been a live interpretative option concerning the nature of the wave function within collapse theories, in particular among those that have defended a primitive ontology view of GRW. Moreover, the dispositions postulated by collapse theories can be defined as propensities, that is dispositions which display probabilistic manifestations. In this way-it is said-we can provide also a dispositional interpretation of the objective probabilities postulated by collapse theories. The problem I stressed in this paper is that, within the literature concerning the nature of the wave function, the ability of dispositions to ground probabilities is merely mentioned and it is never discussed in depth-as far as I know. Frigg and Hoefer [22], while discussing about objective probabilities in GRW, present a sketch of a plausible propensity account for GRW. However, they do not provide a precise definition of those dispositions-contrary to what it is usually required from a metaphysical account of dispositions, which are supposed to entail precise counterfactual conditionals about what the bearer of the disposition would do in certain conditions. In addition, their intuitions concerning the nature of GRW propensities are questionable and the rough account they put forward is problematic, as we can see once we formalize it. On the other hand, Suárez [34] proposes a slightly more formal account of those propensities. However, also his view faces a decisive challenge.

My first aim in this paper has been to formulate a precise inventory of different kinds of propensities, in order to express more precisely the accounts which are present in the literature. Then, among those kinds of dispositions, I identified one kind of propensity which is particularly suitable to account for the probabilistic features of GRW theories. ${ }^{25}$ Those propensities satisfy our desiderata and-following Dorato and Esfeld [15], Esfeld and Gisin [18], Egg and Esfeld [16] and Esfeld et al. [19]can be also defined as what the wave function refers to.

Acknowledgements I am deeply grateful to Claudio Calosi, Christian Wüthrich, Kevin Mulligan, Michael Esfeld, Mauro Dorato, Anna Marmodoro, Francesco Ponti and one anonymous referee for all their useful comments on different versions of this paper.

Funding Open Access funding provided by Università della Svizzera italiana.

\section{Declarations}

Conflict of interest The author declares that they have no conflict of interest to disclose.

\footnotetext{
${ }^{25}$ I want to suggest that they could be extended also to other versions of collapse theories, such as the continuous spontaneous localization model (CSL), if suitably amended.
} 
Open Access This article is licensed under a Creative Commons Attribution 4.0 International License, which permits use, sharing, adaptation, distribution and reproduction in any medium or format, as long as you give appropriate credit to the original author(s) and the source, provide a link to the Creative Commons licence, and indicate if changes were made. The images or other third party material in this article are included in the article's Creative Commons licence, unless indicated otherwise in a credit line to the material. If material is not included in the article's Creative Commons licence and your intended use is not permitted by statutory regulation or exceeds the permitted use, you will need to obtain permission directly from the copyright holder. To view a copy of this licence, visit http://creativecommons.org/licen ses/by/4.0/.

\section{References}

1. Albert, D.Z.: Elementary quantum metaphysics. In: Cushing, J.T., Fine, A., Goldstein, S. (eds.) Bohmian mechanics and quantum theory: an appraisal, pp. 277-284. Springer, Dordrecht (1996)

2. Albert, D.Z.: Wave function realism. In: Ney, A., Albert, D.Z. (eds.) The wave function: essays on the metaphysics of quantum mechanics, pp. 52-57. Oxford University Press, Oxford (2013)

3. Allori, V., Goldstein, S., Tumulka, R., Zanghì, N.: On the common structure of Bohmian mechanics and the Ghirardi-Rimini-Weber theory: dedicated to Giancarlo Ghirardi on the occasion of his 70th birthday. Br J Philos Sci 59(3), 353-389 (2008)

4. Bassi, A., Ghirardi, G.: Collapse theories. In: Zalta, E.N. (ed.) The Stanford encyclopedia of philosophy (Summer 2020 edn). Stanford University, Stanford (2020)

5. Bassi, A., Ghirardi, G.: Dynamical reduction models. Phys. Rep. 379(5-6), 257-426 (2003)

6. Bell, J.S.: Speakable and unspeakable in quantum mechanics: collected papers on quantum philosophy. Cambridge University Press, Cambridge (2004)

7. Belot, G.: Quantum states for primitive ontologists. Eur. J. Philos. Sci. 2(1), 67-83 (2012)

8. Bird, A.: Nature's metaphysics: laws and properties. Oxford University Press, Oxford (2007)

9. Childers, T.: Philosophy and probability. Oxford University Press, Oxford (2013)

10. Chen, E.K.: Realism about the wave function. Philos. Comp. (2019). https://doi.org/10.1111/phc3. 12611

11. Choi, S., Fara, M.: Dispositions. In: Zalta, E.N. (ed.) The Stanford Encyclopedia of Philosophy (Fall 2018 edn). Stanford University, Stanford (2018)

12. Clifton, R., \& Monton, B. (1999). Losing your marbles in wavefunction collapse theories. B r . J. Philos. Sci. 50(4), 697-717.

13. Dekking, F.M., Kraaikamp, C., Lopuhaä, H.P., Meester, L.E.: A modern introduction to probability and statistics: understanding why and how. Springer, New York (2005)

14. Dorato, M.: Dispositions, relational properties and the quantum world. In: Kistler, M., Gnassounou, B. (eds.) Dispositions and causal powers. Ashgate Publishing Ltd, Farnham (2007)

15. Dorato, M., Esfeld, M.: GRW as an ontology of dispositions. Stud. Hist. Philos. Sci. B 41(1), 41-49 (2010)

16. Egg, M., Esfeld, M.: Primitive ontology and quantum state in the GRW matter density theory. Synthese 192(10), 3229-3245 (2015)

17. Esfeld, M., Deckert, D.A.: A minimalist ontology of the natural world. Routledge, Milton Park (2017)

18. Esfeld, M., Gisin, N.: The GRW flash theory: a relativistic quantum ontology of matter in spacetime? Philos. Sci. 81(2), 248-264 (2014)

19. Esfeld, M., Lazarovici, D., Lam, V., Hubert, M.: The physics and metaphysics of primitive stuff. Br. J. Philos. Sci. 68(1), 133-161 (2017)

20. Fetzer, J.H.: Probability and objectivity in deterministic and indeterministic situations. Synthese 57(3), 367-386 (1983)

21. Frigg, R.: GRW theory (Ghirardi, Rimini, Weber model of quantum mechanics). In: Greenberger, D., Hentschel, K., Weinert, F. (eds.) Compendium of quantum physics: concepts, experiments, history and philosophy. Springer, New York (2009)

22. Frigg, R., Hoefer, C.: Probability in GRW theory. Stud. Hist. Philos. Sci. B 38(2), 371-389 (2007)

23. Gebharter, A., Fischer, F.: A causal Bayes net analysis of dispositions. Synthese (2019). https://doi. org/10.1007/s11229-019-02374-8 
24. Gillies, D.: Varieties of propensity. Br. J. Philos. Sci. 51(4), 807-835 (2000)

25. Ghirardi, G.: Sneaking a look at god's cards: unraveling the mysteries of quantum mechanicsrevised edition. Princeton University Press, Princeton (2007)

26. Ghirardi, G.C., Grassi, R. \& Benatti, F. Describing the macroscopic world: Closing the circle within the dynamical reduction program. Found. Phys. 25, 5-38 (1995)

27. Ghirardi, G., Rimini, A., Weber, T.: Unified dynamics for microscopic and macroscopic systems. Phys. Rev. D 34(2), 470 (1986)

28. Hájek, A.: Interpretations of Probability. In: Zalta, E.N. (ed.) The Stanford encyclopedia of philosophy (Fall 2019 edn). Stanford University, Stanford (2019)

29. Mellor, D.H.: The matter of chance. Cambridge University Press, Cambridge (1971)

30. Mellor, D.H.: Probability: a philosophical introduction. Routledge, Milton Park (2004)

31. Miller, D. (1994). Critical rationalism: A restatement and defence. Open Court.

32. Placek, T.: Causal probabilities in GRW quantum mechanics. In: Galavotti, M.C., Dieks, D., Gonzalez, W.J., Hartmann, S., Uebel, T., Weber, M. (eds.) New directions in the philosophy of science, pp. 561-576. Springer, Dordrecht (2014)

33. Popper, K.R.: The propensity interpretation of probability. Br. J. Philos. Sci. 10(37), 25-42 (1959)

34. Suárez, M.: Quantum propensities. Stud. Hist. Philos. Sci. B 38(2), 418-438 (2007)

35. Timpson, C.G.: Probabilities in realist views of quantum mechanics. In: Beisbart, C., Hartmann, S. (eds.) Probabilities in physics. Oxford University Press, Oxford (2011)

Publisher's Note Springer Nature remains neutral with regard to jurisdictional claims in published maps and institutional affiliations. 